\title{
HEAT TRANSFER ANALYSIS OF A SECOND GRADE FLUID OVER A STRETCHING SHEET
}

\author{
C.E. MANESCHY \\ Department of Mechanical Engineering \\ University of Pittsburgh \\ Pittsburgh, PA 15261 \\ M. MASSOUDI \\ U.S.Department of Energy \\ Pittsburgh Energy Technology Center \\ Pittsburgh, PA 15236
}

(Received November 15, 1993 and in revised form January 27, 1994)

\begin{abstract}
The heat tranfer and flow of a non-Newtonian fluid past a stretching sheet is analyzed in this paper. Results in a non-dimensional form are presented here for the velocity and temperature profiles assuming different kind of boundary conditions.
\end{abstract}

KEY WORDS AND PHRASES. Non-Newtonian fluid, Rheology, Viscoelastic liquids. 1980 AMS SUBJECT CLASSIFICATION CODE. 76A05

\section{INTRODUCTION.}

The boundary layer flow of non-Newtonian fluids over a stretching sheet has been studied extensively in the recent years. This problem is of interest when a polymer sheet is extruded continuously from a die. In a recent paper, Maneschy, et al [1] investigated the flow of a second grade fluid over a porous elastic sheet due to stretching and gave a brief review of the previous works. In the present analysis, we extend the result of Maneschy, et al [1] to study the heat transfer over a stretching sheet with suction at the surface. We will first give a brief review of the heat transfer studies for this problem.

Erickson, et al. [2] discussed the heat and mass transfer over a stretching sheet with suction or injection. The fluid was assumed to be an incompressible viscous fluid and the surface speed was assumed to be constant. Later, Gupta and Gupta [3] extended this work by assuming that the surface speed varies with the coordinate along the flow. In both of these investigations, results for velocity, temperature and concentration profiles are presented. Dutta, et al. [4] analyzed the case where a uniform heat flux is prescribed at the surface and the surface is nonporous. Dutta and Gupta [5] also considered the case where the temperature of the sheet is variable. 
While in all these studies the fluid was assumed to be Newtonian. Siddappa and Abel [6] discussed this problem assuming a viscoelastic fluid with suction at the surface. They obtained analytic solution for the velocity and temperature fields. The effects of viscous dissipation were ıgnored in this paper. Bujurke, et al. [7] assumed cubic profiles for velocity and temperature fields and used a second order fluid model. They, however, did not consider thermodynamical restrictions imposed by the Clausius-Duhem inequality (cf. Dunn and Fosdick [8]). Char and Chen [9] considered the fluid to be of Walters' liquid B model and the plate was subjected to variable heat flux. Dandapat and Gupta [10] presented a critical review of previous works where the fluid had been assumed to be non-Newtonian. In particular, they showed the errors and inconsistencies in the works of Siddappa and Abel [6] and Bujurke, et al. [7]. However, the analysis of Dandapat and Gupta [10] is also incomplete in the sense that (i) no thermodynamical considerations are taken into account, and (ii) the effect of viscous dissipation is neglected. While the later is justifiable as an assumption, the former could be considered as a serious flaw in the analysis. Two other recent works which need to be mentioned are those of Surma Devi and Nath [11] and Lawrence and Rao [12]. In the first case, the fluid was assumed to be a second grade electrically conducting fluid. Lawrence and Rao [12] basically considered the same problem as Dandapat and Gupta [10], except that they give more detailed solution of the velocity and temperature fields, for different values of the non-Newtonian parameter.

In this paper, we assume the fluid is thermodynamically compatible in the sense that it satisfies the Clausis-Duhem inequality. The momentum equation is solved using the numerical method discussed in Maneschy, et al. [1] and the results are then used to find the temperature distribution in the boundary layer.

\section{GOVERNING EQUATIONS.}

We consider the flow of a second-grade fluid past a porous stretching sheet coinciding with the plane $y=0$ and subjected to suction at this plane. Two equal and opposite forces are applied along the $x$-axis in such a way that the origin remains fixed and the velocity of stretching is proportional to the distance from the origin. The sheet is assumed to have constant temperature $T_{w}$ at its surface and $T_{\infty}$ is the temperature of the ambient fluid. Under these assumptions, the boundary layer equations governing the flow can be written as

$$
\begin{aligned}
& \frac{\partial u}{\partial x}+\frac{\partial v}{\partial y}=0 \\
& u \frac{\partial u}{\partial x}+v \frac{\partial v}{\partial y}=v \frac{\partial^{2} u}{\partial y^{2}}+k_{1}\left[\frac{\partial}{\partial x}\left(u \frac{\partial^{2} u}{\partial y^{2}}\right)+\frac{\partial u}{\partial y} \frac{\partial^{2} v}{\partial y^{2}}+v \frac{\partial^{3} u}{\partial y^{3}}\right] \\
& u \frac{\partial T}{\partial x}+v \frac{\partial T}{\partial y}=K \frac{\partial^{2} T}{\partial y^{2}}
\end{aligned}
$$

with

$$
v=\mu / \rho ; \quad k_{1}=\alpha_{1} / \rho
$$

where $\mu$ is the coefficient of viscosity, $\rho$ the density of the fluid, $K$ the thermal conductivity and $\alpha_{1}$ 
the material modulus usually referred to as the first normal stress coefficient. The effects of viscous dissipation are neglected in equation (2.3).

The sign of the parameter $\alpha_{1}$, and therefore $k_{1}$ in equation (2.2), has been the subject of much controversy. Thermodynamic and compatibility in a sense that all motions of the fluid meet the Clausius-Duhem inequality and the assumption that the specific Helmholtz free energy of the fluid be a minimum when the fluid is locally at rest require, among other conditions, that $\alpha_{1}>0$. Although experiments carried out in many dilute polymeric liquids, under the assumption that the fluid is second-grade, seem to imply that $\alpha_{1}<0$, studies by Dunn and Fosdick [8] and Fosdick and Rajagopal [13] show that when this is the case the rest state of the fluid is unstable or the fluid exhibits properties that are clearly physically unacceptable. Without getting into a detailed discussion of this matter, we will assume that the normal stress coefficient $\alpha_{1}$ is positive throughout this analysis. Readers should refer to a recent exhaustive critical review by Dunn and Rajagopal [14] on the thermomechanics of fluid of differential type.

The boundary conditions for this problem are

$$
\begin{aligned}
& u=C x ; v=-V_{0} ; T=T_{w} \text { at } y=0, \quad C>0 \\
& u \rightarrow 0 ; T \rightarrow T_{\infty} \text { as } y \rightarrow \infty,
\end{aligned}
$$

where $V_{0}$ is the suction velocity at the plate surface.

Let us introduce the following dimensionless variables

$$
\bar{x}=\frac{x}{L} ; \quad \bar{y}=\frac{y}{L} R^{1 / 2} ; \bar{u}=\frac{u}{C L} ; \bar{v}=\frac{v}{C L} R^{1 / 2} ; R=\frac{C L^{2}}{v} ; \theta=\frac{T-T_{w}}{T_{\infty}-T_{w}},
$$

where $R$ is the Reynolds number for the flow and $L$ is the characteristic length. If we further define the non-dimensional velocity components as

$$
\bar{u}=\bar{x} f^{\prime}(\bar{y}) ; \quad \bar{v}=-f(\bar{y}),
$$

then it can be shown that equations (2.1)-(2.3) reduce to

$$
\begin{aligned}
& f^{\prime 2}-f f^{\prime \prime}=f^{\prime \prime \prime}+k\left[2 f^{\prime} f^{\prime \prime \prime}-f^{\prime 2}-f f^{\prime V}\right], \\
& \theta^{\prime \prime}+\operatorname{Pr} f \theta^{\prime}=0,
\end{aligned}
$$

where

$$
k=\frac{k_{1}}{L^{2}} R ; \quad \operatorname{Pr}=\frac{v}{K},
$$

and the prime denotes differentiation with respect to $\bar{y}$. In deriving (2.9) the temperature profile was assumed in the form $\bar{\theta}=\bar{\theta}(\bar{y})$. The boundary conditions are

$$
\begin{aligned}
& f=-\frac{V_{0}}{C L} R^{1 / 2} ; f^{\prime}=1 ; \theta=0 \text { at } \bar{y}=0 \\
& f^{\prime} \rightarrow 0 ; \theta \rightarrow 1 \text { as } \bar{y} \rightarrow \infty .
\end{aligned}
$$


It is important to note that equation (2.8), (2.10) and (2.11) represent a two-point non-linear fourth-order differential equation having only three boundary conditions and, therefore, an additional condition for the function $f$ is required. Since in general far away from the plate the flow is uniform, Rajagopal and Gupta [15] suggested using the condition that

$$
\frac{\partial \bar{u}}{\partial \bar{y}} \rightarrow 0 \quad \text { or } \quad f^{\prime \prime} \rightarrow 0 \quad \text { as } \quad \bar{y} \rightarrow \infty .
$$

\section{NUMERICAL SOLUTION.}

Equations (2.8) and (2.9) constitute the final system of differential equations to be solved. First, the non-linear equation (2.8) is solved for the velocity function $f(\bar{y})$ with the result, in turn, substituted in (2.9) to determine the temperature distribution $\theta(\bar{y})$. Following the quasi-linearization method developed by Bellman and Kalaba [16], equation (2.9) can be written in the form below

$$
\begin{aligned}
& f^{\prime v}-f^{\prime \prime \prime}\left(2 \bar{f}^{\prime}+\frac{1}{k}\right) \frac{1}{\bar{f}}-f^{\prime \prime}\left(\frac{1}{k}-\frac{2 \bar{f}^{\prime \prime}}{\bar{f}}\right)-\frac{2 f^{\prime}}{\bar{f}}\left(\bar{f}^{\prime \prime \prime}-\frac{\bar{f}^{\prime}}{k}\right) \\
& -\frac{f}{\bar{f}^{2}}\left[\bar{f}^{\prime \prime 2}-2 \bar{f}^{\prime \prime \prime} \bar{f}^{\prime}+\frac{\bar{f}^{\prime 2}}{k}-\frac{\bar{f}^{\prime \prime \prime}}{k}\right]=\frac{\bar{f}^{\prime \prime \prime}}{k \bar{f}}
\end{aligned}
$$

where $f$ and $\bar{f}$ represent the current and previous approximation to the solution, respectively.

Equation (3.1) is a linear non-homogeneous equation in the unknown function $f$. Its solution can be written as a linear combination of two linear independent solutions to the homogeneous problems, $f_{H 1}$ and $f_{H 2}$, and a particular solution, $f_{p}$, such that

$$
f=C_{1} f_{H 1}+C_{2} f_{H 2}+f_{p}
$$

In order to satisfy boundary conditions (2.10) and (2.11) and the linear independence requirement, the three solutions above are assumed to have the following values at $y=0$

$$
\begin{aligned}
& {\left[f_{H 1}, f_{H 1}^{\prime}, f_{H 1}^{\prime \prime}, f_{H 1}^{\prime \prime \prime}\right]=(0,0,1,0)} \\
& {\left[f_{H 2}, f_{H 2}^{\prime}, f_{H 2}^{\prime \prime}, f_{H 2}^{\prime \prime \prime}\right]=(0,0,0,1)} \\
& {\left[f_{p}, f_{p}^{\prime}, f_{p}^{\prime \prime}, f_{p}^{\prime \prime \prime}\right]=\left(\frac{-V_{0}}{C L} R^{1 / 2}, 1,0,0\right) .}
\end{aligned}
$$

For each of the homogeneous and particular solutions, equation (3.1) is numerically integrated to $y=y_{\infty}$ using the Runge-Kutta integration method. The values found at the end point are used to find the pair of constants $C_{1}$ and $C_{2}$ so that the boundary conditions at $y=y_{\infty}$ are met. Therefore,

$$
\begin{aligned}
& C_{1} f_{H 1}^{\prime}\left(y_{\infty}\right)+C_{2} f_{H 2}^{\prime}\left(y_{\infty}\right)+f_{p}^{\prime}\left(y_{\infty}\right)=0 \\
& C_{2} f_{H 1}^{\prime \prime}\left(y_{\infty}\right)+C_{2} f_{H 1}^{\prime \prime}\left(y_{\infty}\right)+f_{p}^{\prime}\left(y_{\infty}\right)=0 .
\end{aligned}
$$

Once the values for the constants are found the function $f$ can be obtained from equation (3.2) for all discretization points $0=y_{1}<\ldots<y_{J}<\ldots<y_{N}=y_{\infty}$. This process is repeated until the 
magnitude of the difference between two consecutives iterations fall below a prescribed tolerance, at all points. In this work, a tolerance limit of $10^{-7}$ was used. As the zeroth approximation to the solution, the function

$$
f_{0}(y)=y,
$$

satısfying all boundary conditions, was assumed.

The solution for the function $f$ is substituted in equation (2.9), which is then solved using the finite difference technique for the temperature profile.

\section{RESULTS AND DISCUSSIONS}

The results obtained by solving the differential equations derived in the previous sections will be now outlined for different values of the folowing parameters

$$
k=\frac{\alpha_{1} R}{\rho L^{2}} ; \quad a=\frac{V_{0}}{C L} R^{1 / 2} ; \quad \operatorname{Pr}=\frac{v}{K}
$$

For a given flow, these parameters account for the effects of the normal stress coefficient, $\alpha_{1}$, the suction velocity, $V_{0}$, and the Prandtl number, $\mathrm{Pr}$, on the solution.

The value of $f$ as a function of $y$ is plotted in figure 1 for different values of $k$, assuming nosuction at the plate wall $(a=0)$. This function represents the normal component of the velocity for the flow. As it can be seen from figure 1, the magnitude of the normal velocity component increases significantly for high values of the normal stress coefficient, $\alpha_{1}$.

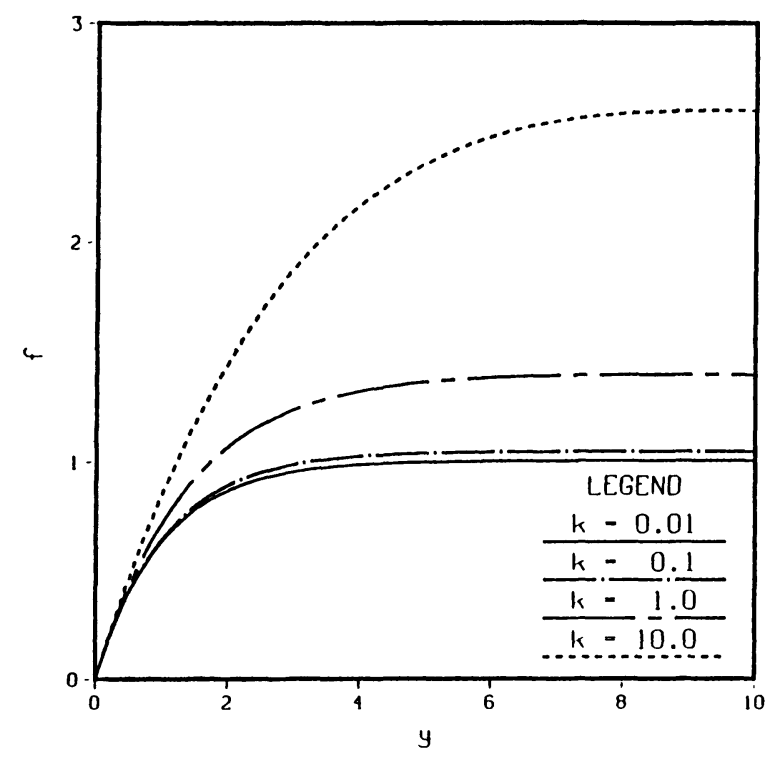

Fig. 1. Function $f$ for Different Values of $k$.

Figure 2 indicates the temperature variation when $\operatorname{Pr}=1.0$ and $a=0$, for different values of $k$. It can be seen that increasing the normal stress coefficient has the effect of decreasing the thermal boundary layer thickness. Figure 3 displays the temperature profiles for different values of $\operatorname{Pr}$, 
assuming $k=1.0$ and no suction at the wall $(a=0.0)$. As is expected, for higher values of the Prandtl number, there is a decrease in the thermal boundary layer. The variation of the temperature with the suction velocity is shown in figure 4 for $k=1.0$ and $\operatorname{Pr}=1.0$. Similar to the results observed in the other two cases, as the suction velocity increases the thermal boundary layer decreases.

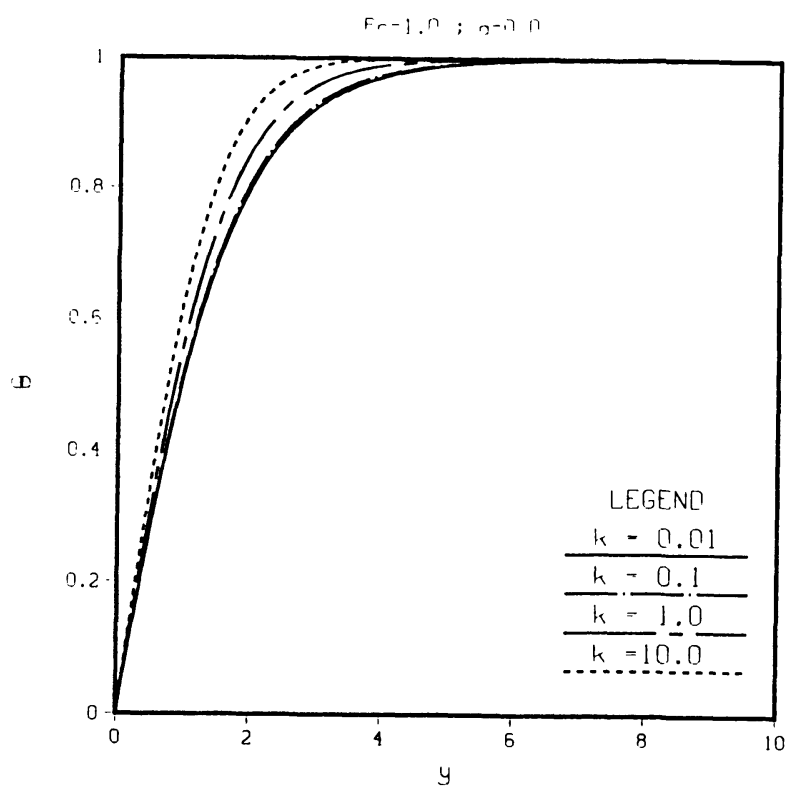

Fig. 2. Temperature Profile as Function of Non-Newtonian Coefficient.

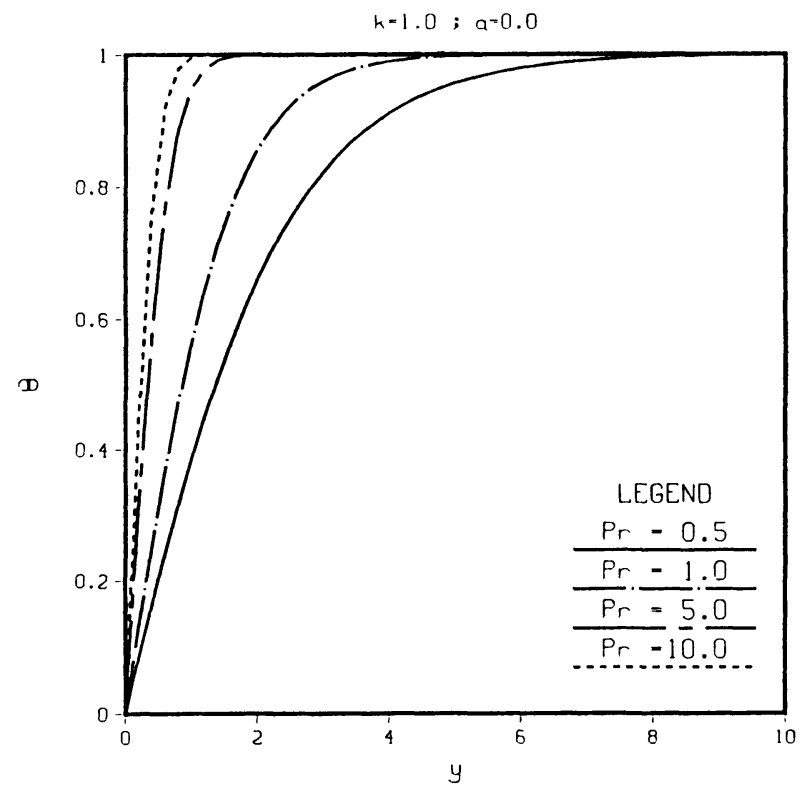

Fig. 3. Temperature Profile as Function of Prandtl Number. 


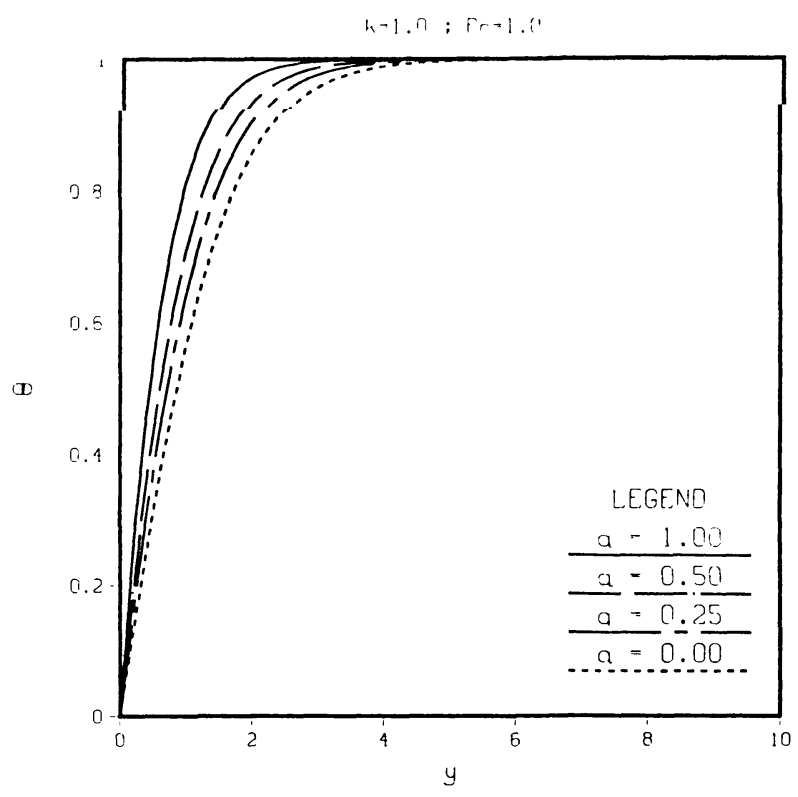

Fig. 4. Temperature Profile as Function as Suction Velocity.

\section{REFERENCES}

1. Maneschy, C.E., M.Massoudi and K.R.Rajagopal, "Flow of a second grade fluid over a porous elastic sheet due to stretching," To appear in the Journal of Mathematical and Physical Science (1993).

2. Erickson, L.E., L.T.Fan and V.G.Fox, "Heat and mass transfer on a moving continuous flat plate with suction or injection," I\&EC Fund., Vol.5 (1966), p.19.

3. Gupta, P.S. and A.S.Gupta, "Heat and mass transfer on a stretching sheet with suction or blowing," Canadian J. Chem. Engng., Vol.55 (1977), p.744

4. Dutta, B.K., P.Roy and A.S.Gupta, "Temperature field in flow over a stretching sheet with uniform heat flux," Int. Comm. Heat Mass Transfer, Vol.12 (1985), p.89.

5. Dutta, B.K. and A.S.Gupta, "Cooling of a Stretching sheet in a viscous flow," Ind. Eng.Chem. Res., Vol.26 (1987), p.333.

6. Siddappa, B. and M.S.Abel, "Visco-elastic boundary layer flow past a stretching plate with suction and heat transfer," Rheol. Acta, Vol.25 (1986), p.319.

7. Bujurke, N.M, S.N.Biradar and P.S.Hiremath, "Second-order fluid flow past a stretching sheet with heat transfer," ZAMP, VOL.38 (1987), p.654.

8. Dunn, J.E. and R.L.Fosdick, "Thermodynamics, stability, and boundeness of fluids of complexity 2 and fluids of second grade," Arch. Rat. Mech. Anal., Vol.56 (1974), p.191. 
9. Char, M.I. and C.K.Chen, "Temperature field in non-Newtonian flow over a stretching plate with variable heat flux," Intl. J. Heat Mass Transfer, Vol.31 (1988), p.917.

10. Dandapat, B.S. and A.S.Gupta, "Flow and heat transfer in a viscoelastic fluid over tretching sheet," Int. J. Non-Linear Mech., Vol.24 (1989), p.215.

11. Surma Devi, C.D. and G.Nath, "Flow and heat transfer of a viscoelastic fluid over a stretching sheet with variable wall temperature or heat flux," Indian J. Tech., Vol.28 (1990), p.93.

12. Lawrence, P.S. and B.N.Rao, "Heat transfer in the flow of a viscoelastic fluid over a stretching sheet," Acta Mech., Vol.93 (1992), p.53.

13. Fosdick, R.L. and K.R.Rajagopal, "Anomalous features in the model of second order fluids," Arch. Rat. Mech. Anal., Vol.70 (1978), p.145.

14. Dunn, J.E. and K.R.Rajagopal, "A critical historical review and thermodynamics analysis of fluids of differential type," Submitted for publication (1993).

15. Rajagopal, K.R. and A.S.Gupta, "An exact solution for the flow of a non-Newtonian fluid past an infinite porous plate," Meccanica, Vol.19 (1984), p.158.

16. Bellman, R.E. and R.E.Kalaba, "Quasilinearization and non-linear boundary value problems," American Elsevier, New York (1965). 


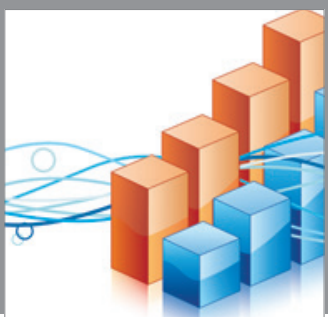

Advances in

Operations Research

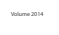

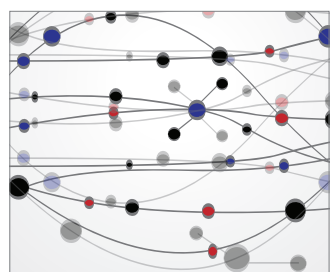

\section{The Scientific} World Journal
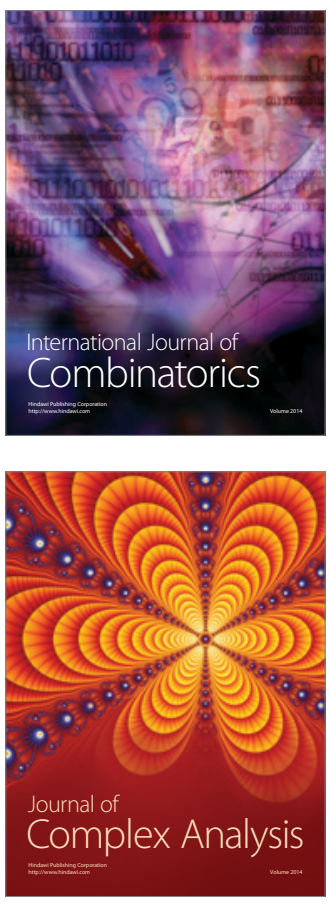

International Journal of

Mathematics and

Mathematical

Sciences
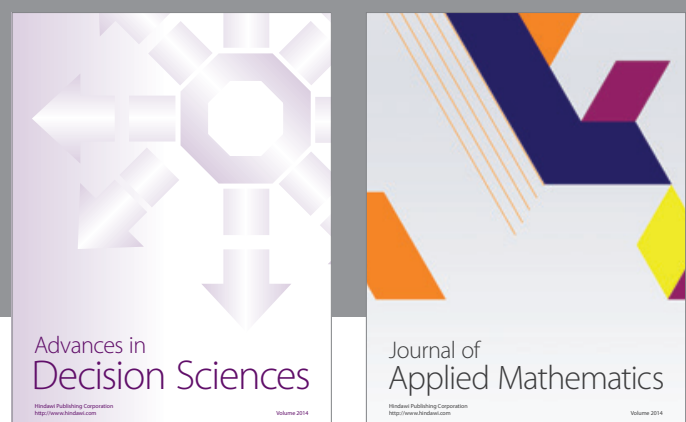

Journal of

Applied Mathematics
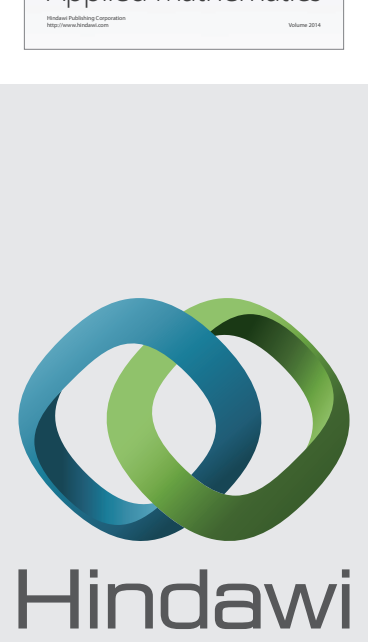

Submit your manuscripts at http://www.hindawi.com
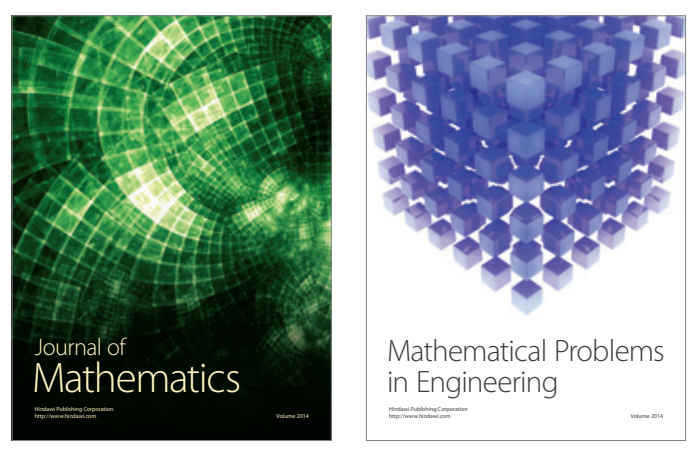

Mathematical Problems in Engineering
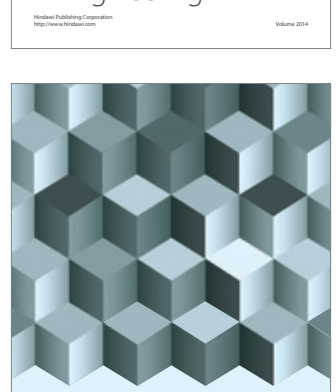

Journal of

Function Spaces
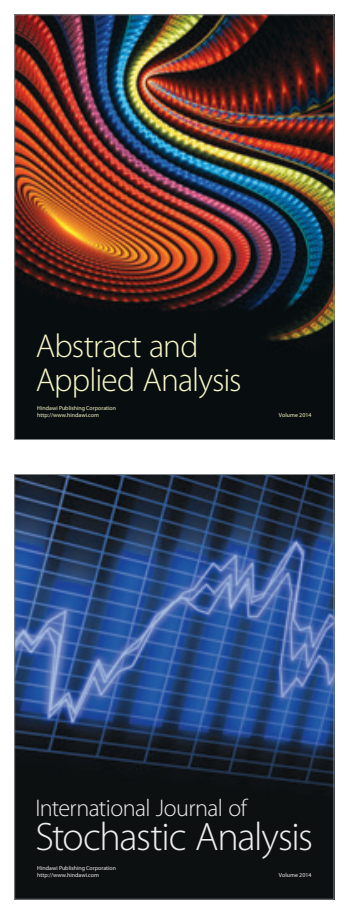

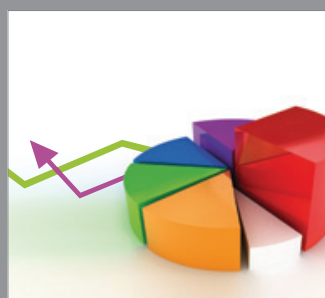

ournal of

Probability and Statistics

Promensencen
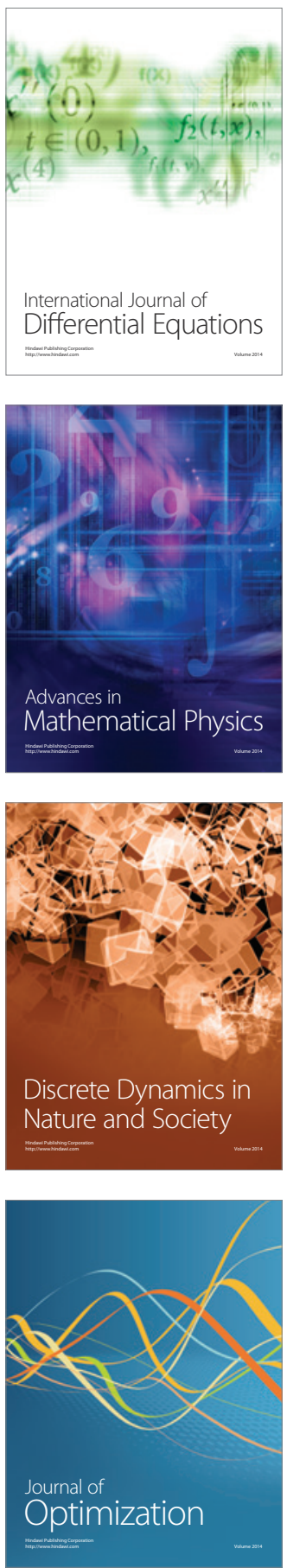